\title{
Research Paper A new approach of ratio estimation in sample surveys
}

\author{
- M. IQBAL JEELANI, S.E.H. RIZVI, MANISH KR. SHARMA, NAGEENA \\ NAZIR AND FAHEEM JEELANI
}

See end of the paper for authors' affiliations

Correspondence to : M IQBAL JEELANI Sher-e-Kashmir University of Agricultural Sciences and Technology of Jammu, JAMMU (J\&K) INDIA

Email : jeelani.miqbal@

gmail.com

Paper History :

Received : 05.01.2017;

Revised : 26.01.2017;

Accepted : 05.02.2017
Aвstract : This article deals with the estimation of population mean under simple random sampling using a new form of ratio estimator. The expression for mean square error and bias has been obtained. An efficiency comparison is considered for proposed estimator with the classical ratio, product and exponential ratio estimator. Finally an empirical study is also carried out to judge the performance of proposed estimator.

KEY Words : Simple random sampling, Ratio estimator, Mean square error, Efficiency, AMS Classification: 62D05

How To Cite This Paper : Jeelani, M. Iqbal, Rizvi, S.E.H., Sharma, Manish Kr., Nazir, Nageena and Jeelani, Faheem (2017). A new approach of ratio estimation in sample surveys. Internat. Res. J. Agric. Eco. \& Stat., 8 (1) : 100-103, DOI : 10.15740/HAS/IRJAES/8.1/100-103.

\section{INTRODUCTION :}

In sampling it is a well known fact that the proper use of auxiliary information supplied by auxiliary variable improves the efficiency of the estimators of population parameters of the study variable. Ratio estimation is one such example where this criterion is fulfilled. Ratio estimators are used for estimation of population mean when study variable and auxiliary variable are highly correlated with each other. Early historical developments of the ratio method of estimation are being well presented by, Sen (1993). Some of the modified ratio estimators given by Cochran (1977); Murthy (1967); Prasad (1989); Sen (1993); Singh and Tailor (2003 and 2005); Upadhyaya and Singh (1999); Yan and Tian (2010) and Jeelani and Maqbool (2013) are available in literature.

The classical ratio estimator for population mean $\bar{Y}$ of the study variable is defined as $\overline{\mathbf{y}}_{\mathbf{r}}=\overline{\mathbf{y}} / \mathbf{\mathbf { x }} \overline{\mathbf{X}}=\hat{\mathbf{R}} \overline{\mathbf{X}}$ assuming that population mean of the auxiliary variable is known $\bar{y}$ and $\bar{x}$ are sample means of study variable and auxiliary variable, and the mean square of the classical ratio estimator is $\left(\mathbf{M S E}\left(\overline{\mathbf{y}}_{\mathbf{r}}\right)=\delta \overline{\mathbf{Y}}^{\mathbf{2}}\left[\mathbf{C}_{\mathbf{y}}^{2}+\mathbf{C}_{\mathbf{x}}^{\mathbf{2}}-\mathbf{2} \rho_{\mathbf{y x}} \mathbf{C}_{\mathbf{y}} \mathbf{C}_{\mathbf{x}}\right]\right)$. Where $\delta=1-\mathbf{f} / \mathbf{n}, \mathbf{f}=1 / \mathbf{n}, \hat{\mathbf{R}}$ population ratio, $\rho_{\mathrm{yx}}$ is correlation coefficient between study variable and auxiliary variable, $\mathrm{C}_{\mathrm{y}}$ and $\mathrm{C}_{\mathrm{x}}$ are co-efficient of variations of study variable and auxiliary variable. Also $\mathbf{C}_{\mathbf{y}}=\frac{\mathbf{S}_{\mathbf{y}}}{\overline{\mathbf{Y}}}, \mathbf{C}_{\mathbf{x}}=\frac{\mathbf{S}_{\mathbf{x}}}{\mathbf{X}}, \boldsymbol{\rho}_{\mathbf{y x}}=\frac{\mathbf{S}_{\mathbf{y x}}}{\mathbf{S}_{\mathbf{y}} \mathbf{S}_{\mathbf{x}}}$, respectively.

\section{Suggested estimator:}

In this section we suggest a new ratio estimator by 